\title{
Dynamic adaptation of personal ubicomp environments
}

\author{
Rui S. Moreira ${ }^{1,2} \cdot$ José Torres $^{1} \cdot$ Pedro Sobral $^{1} \cdot$ Ricardo Morla $^{2,3}$. \\ Mark Rouncefield ${ }^{4}$. Gordon S. Blair ${ }^{4}$
}

Published online: 10 March 2016

(C) Springer-Verlag London 2016

\section{Introduction}

A significant challenge for personal and ubiquitous computing is to cope with frequent changes of user preferences, profile, location, and context in general. In this special issue we bring you an update on how dynamic adaptation is being used to address this challenge. Dynamic adaptation provides runtime modeling and monitoring of both structural and behavioral aspects, possibly at different levels of the open systems interconnection (OSI) model. We are thinking in particular of personal and ubiquitous computing environments assembled from a variety of custom-of-theshelf (COTS) systems that are planned to work independently of each other. Dynamic adaptation opens the door to

Rui S. Moreira

rmoreira@ufp.edu.pt

José Torres

jtorres@ufp.edu.pt

Pedro Sobral

pmsobral@ufp.edu.pt

Ricardo Morla

rmorla@fe.up.pt

Mark Rouncefield

m.rouncefield@comp.lancs.ac.uk

Gordon S. Blair

gordon@comp.lancs.ac.uk

1 Faculdade de Ciências e Tecnologia, Universidade Fernando Pessoa, Porto, Portugal

2 INESC TEC, Laboratório Associado, Porto, Portugal

3 Faculdade de Engenharia, Universidade do Porto, Porto, Portugal

4 Faculty of Applied Sciences, Lancaster University, Lancaster, UK reason about and undertake policies and strategies without compromising the functionality and integration of COTS systems in the environment. The papers in this special issue cover different aspects of dynamic adaptation in PUC.

\section{Contributions}

The first paper is entitled "A model for learning objects adaptation in light of mobile and context-aware computing" and provides motivation for our topic with an example of using adaptation in an educational setting. The authors of this paper present EduAdapt, an architectural model for the adaptation of learning multimedia objects according to mobile device characteristics, learning style, and other context information from the students. To make this adaptation the authors use inference and rules in their proposed OntoAdapt ontology. This ontology is used to describe context, learner profile, learning style, and device characteristics and its purpose is to help with the recommendation of learning objects to students and the adaption of these objects according to context. This ontology is the core of a mobile learning environment that can adapt contents considering context information and can thus provide a mechanism that assists in the inference of learning objects appropriate for the learner.

The second and third papers show how dynamic adaptation can help address the challenge of frequent changes in well-established research domains common to many personal and ubiquitous computing systems such as the graphical user interface and user security and authentication. The authors of "Declarative GUI descriptions for device-independent applications" give us a critical perspective about existing solutions for user interface adaptation middleware. They start by employing eight popular 
GUI user interface description languages (UIDLs) to describe a set of GUI views for an existing mobile application. They assess qualitative and quantitative criteria for composed GUI views and individual GUI components and conclude that existing declarative UIDLs are not capable of describing mobile GUI in a device-independent manner. They then propose guidelines for improving device-independent UIDL that focus for example on display pixel density, external triggers for user interaction handlers, layout flexibility, and low level graphical primitives. The authors of "Model for adaptable context-based biometric authentication for mobile devices" start by arguing that the most accurate way to perform user authentication depends on the user context. They then propose a model that constitutes a foundation for building adaptable and extensible multifactor context-dependent systems for mobile authentication. Their approach considers the user context in order to dynamically select the most accurate biometric authentication method and also taking into account user's intended interaction scheme at each moment.

Finally, the fourth and fifth papers explore the integration of different PUC systems into the same PUC environment. The fourth paper looks specifically at integration of communications and is entitled "A user-centric approach to dynamic adaptation of reusable communication services." The authors present the design of an autonomic network communication broker that supports the use of multiple communication frameworks and an approach for dynamic adaptation of the communication services with the aim of reducing the complexity of reusing multiple communication frameworks. The paper concludes with a performance evaluation to demonstrate the advantages of their approach over existing systems. The fifth and last paper of this special issue approaches the integration issue from a network management and machine learning perspectives and is entitled "A behavioral reflective architecture for managing the integration of personal ubicomp systems: automatic SNMP-based discovery and management of behavior context in smart-spaces." The authors start by extending the use of existing network management protocols by exposing state and state transition statistics of the different PUC systems in the environment. As this solution relies on a system state specification being provided by manufacturers, the paper then proposes two machine learning approaches for automatic system state discovery. A home health-care and entertainment environment motivates the development and evaluation of these contributions.

Acknowledgments We would like to thank the authors of these papers for their contributions, the reviewers for their willingness to serve in this special issue, and the editors of Personal and Ubiquitous
Computing for giving us this opportunity to report on the current developments in dynamic adaptation for PUC environments. We expect this topic to continue to have interesting developments in the near future.

\section{Appendix: International program committee}

\begin{tabular}{|c|c|}
\hline Adrian Clear & Lancaster University \\
\hline Alvaro Barradas & Universidade Algarve \\
\hline Alvaro Rocha & Universidade Coimbra \\
\hline Anders Andersen & Tromso University \\
\hline António Pereira & Instituto Politecnico Leiria \\
\hline Arnor Solberg & SINTEF \\
\hline Claudio Geyer & Universidade Federal Rio Grande Sul \\
\hline Daniel Goncalves & INESC ID \\
\hline Fabio Costa & Universidade Federal de Goiás \\
\hline Gang Huang & Pekin University \\
\hline Hui Song & SINTEF \\
\hline Isidro Calvo & Universidad Pais Vasco \\
\hline João Correia Lopes & Faculdade Engenharia Universidade Porto \\
\hline Johann Bourcier & IRISA \\
\hline Keith Cheverst & Lancaster University \\
\hline Luis Bernardo & Faculdade Ciencias, Universidade Lisboa \\
\hline Luis Paulo Reis & Universidade Minho \\
\hline Luis Veiga & INESC ID \\
\hline $\begin{array}{l}\text { Manuel Caeiro } \\
\text { Rodriguez }\end{array}$ & Universidade Vigo \\
\hline Mario Kolberg & Stirling University \\
\hline Mario Trapp & IESE Fraunhofer \\
\hline Mattias Rost & University of Glasgow \\
\hline Nelly Bencomo & Aston University \\
\hline Nilanjan Banerjee & University of Maryland \\
\hline Nuno Lau & Universidade de Aveiro \\
\hline Nuno Vasco Lopes & Universidade Minho \\
\hline Paolo Bellavista & Universita di Bologna \\
\hline Paul Grace & Southampton University \\
\hline Paula Prata & Universidade Beira Interior \\
\hline Paulo Fazendeiro & Universidade Beira Interior \\
\hline Paulo Pinto & Faculdade Ciencias, Universidade Lisboa \\
\hline Peter Thomas & PUC Springer Journal \\
\hline Phillip Benachour & Lancaster University \\
\hline Romain Rouvoy & $\begin{array}{l}\text { Laboratoire d'Informatique Fondamentale } \\
\text { de Lille }\end{array}$ \\
\hline Rui Jose & Universidade Minho \\
\hline Thais Batista & $\begin{array}{l}\text { Universidade Federal do Rio Grande do } \\
\text { Norte }\end{array}$ \\
\hline Tiago Guerreiro & Faculdade Ciencias, Universidade Lisboa \\
\hline Yunchuan Sun & Beijing Normal University \\
\hline
\end{tabular}

